\title{
Measurements of lateral ion transport in LCD cells
}

\author{
Goran Stojmenovik $^{\text {a) }}$, Kristiaan Neyts ${ }^{\text {a) }}$, Stefaan Vermael ${ }^{\text {a) }}$, Alwin Verschueren ${ }^{\text {b) }}$, Rob Van \\ Asselt ${ }^{c)}$, Chris Desimpel ${ }^{\text {a) }}$ and Artur Adamski ${ }^{\text {a) }}$
}

\begin{abstract}
The ions present in liquid crystal devices modulate the applied electric field and lead to deterioration of the expected good optical response. In addition to the flicker and ghost images, a boundary image-retention effect is also possible. It occurs near the edges of a stressed pixel. We have attributed this effect to ions moving in the lateral direction, perpendicular to the applied electric field. This hypothesis has been proven using a combination of electrical and optical measurements and the measurements explained using the new model of lateral ion transport. The physical cause of this phenomenon is still not clear and is a subject of further study.
\end{abstract}

Keywords $\square$ Liquid crystal displays; Image retention; Ions; Lateral ion transport

\section{INTRODUCTION}

It is widely known that liquid crystal devices contain ions, whose origin can be traced to impurities due to the fabrication process, to the alignment layers or to the dissociation of the LC mixture molecules. The presence of ions modulates the applied electric field and deteriorates the good electro-optical behavior of the LC cell, leading to flicker and/or image sticking [1-4]. The ion motion and image retention phenomena have widely been investigated, but only in the direction of the applied field, independently on the lateral dimensions along the surface of the cell. We have observed a special form of image retention, which occurs on the boundaries of the stressed pixel. Our highly contaminated test cell had one row $\left(2 \times 3 \mathrm{~cm}^{2}\right)$ and three columns $\left(2 \times 1 \mathrm{~cm}^{2}\right)$. After a longer stressing of the middle column, image retention has developed at the borders with the neighboring columns (Fig. 1).

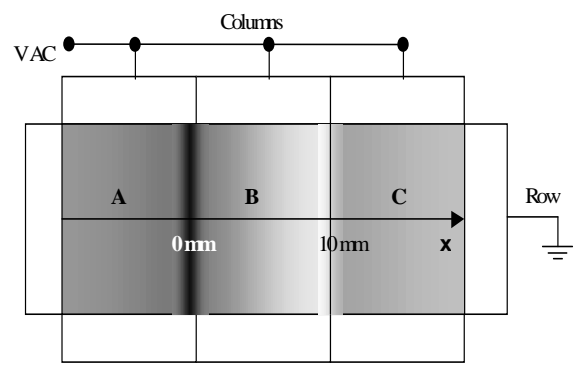

Fig. 1. A scheme of the test cell and the observed phenomenon of image retention in the lateral direction $x$ (viewed at $1.65 \mathrm{~V}, 20 \mathrm{~Hz}$ SQW)

When all columns were viewed at a gray-level voltage and a frequency of $10-50 \mathrm{~Hz}$, there appears a dark stripe on one edge and a bright stripe on the opposite edge (the cell is normally white). The aim of this paper is to show that this

a) LCD group, ELIS, Ghent University, Gent, Belgium, e-mail: goran@elis.rug.ac.be

b) Philips Research Laboratories, Eindhoven, The Netherlands

c) Philips Centre for Industrial Technology, Eindhoven, The Netherlands boundary image retention is caused by lateral movement of ions, a new phenomenon not previously reported.

\section{EXPERIMENTAL METHOD}

At a given gray-level voltage and a low frequency, the movement of the ions influences the optical response of the cell and causes flicker. Using the transmission through the cell, it is possible to determine a qualitative measure for the local ion density. This provides us with a tool to investigate the lateral non-homogeneity in the stressed cell.

Let a square wave (SQW) be applied to a cell. When the pulse with one polarity starts, the ions move towards the oppositely-charged electrodes. If their concentration is high enough, they create a significant inner electric field opposed to the applied field. When the voltage is reversed, at first the electric field in the cell is reinforced. Then the ions again start moving to the opposite electrodes, creating an opposite field as they gather on the other side of the cell. If the voltage is kept constant at the electrodes (direct drive), the change of the voltage over the LC cell during one frame-time is

$$
\Delta V_{L C} \approx \frac{2 e S d_{L C}}{C_{2 A L}} n_{0}
$$

$e$ is the elementary charge, $n_{0}$ - ion concentration, $S$ - cell surface, $d_{L C}-$ cell thickness, $C_{2 A L}$ - alignment layers capacity.

As shown in fig. 2, from the measured transmission modulation within a frame and the transmission-voltage characteristic, we can derive the change of effective voltage over the measured pixel. This change is comparable to $\Delta V_{L C}$ in equation (1), and so we can determine the ion content at the measured spot.

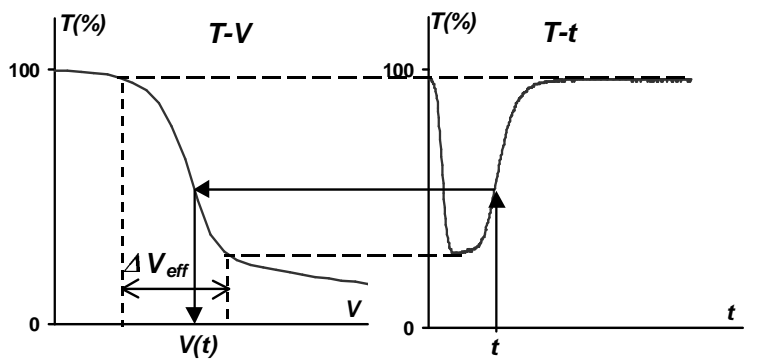

Fig. 2. Illustration of the method used to derive the effective voltage in the cell from transmission measurements

\section{STRESSING, MEASUREMENTS AND ObSERVATIONS}

In order to study the lateral image retention, a fresh test cell with high ion contamination was prepared. The middle column $\mathrm{B}$ was stressed with a square wave (SQW), $2 \mathrm{~V}_{\mathrm{ampl}}$ and $100 \mathrm{~Hz}$, for 3.5 hours, while the side columns were not connected. Before the stress the transmission-voltage curve at $20 \mathrm{~Hz}$ and flicker (transmission-time, T-t) at $1 \mathrm{~Hz}$ and $20 \mathrm{~Hz}$, 
$1.65 \mathrm{~V}$ were measured for lateral points at $0.5 \mathrm{~mm}$ spacing. After each $30 \mathrm{~min}$ stress the $T-t 1 \mathrm{~Hz}$ and $20 \mathrm{~Hz}, 1.65 \mathrm{~V}$ were measured again for each point. We observed a change in the average transmission through the cell as stress time increased (fig. 3)

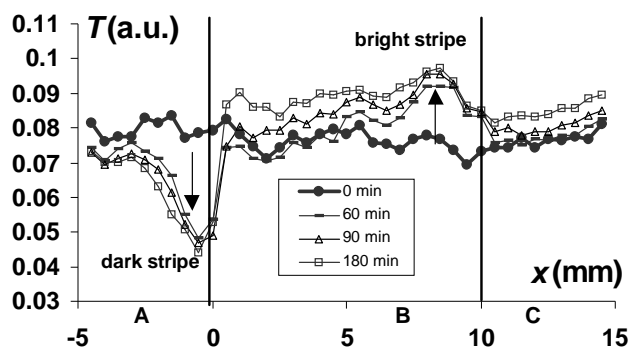

Fig. 3. The occurrence of bright and dark bands in the cell. The average transmission on $1.65 \mathrm{~V}, 20 \mathrm{~Hz}$ changes with increasing the stress time.

In addition, the transient electric current in all the blocks before and after stress has been measured. The total charge in the measured block is obtained from the integral of the current. In fig. 4 we see that the charge in the stressed column $\mathrm{B}$ has decreased, while in the column A to the left has increased after the stress.

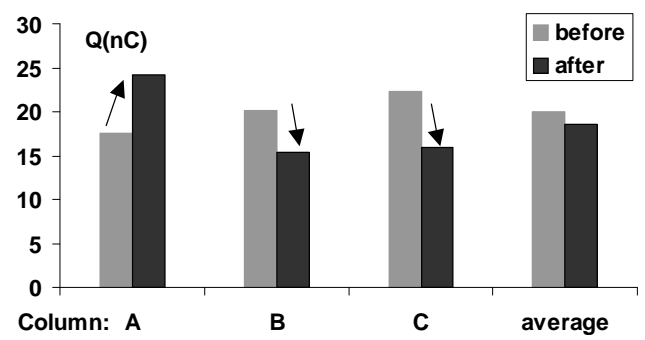

Fig. 4. The total charge in the stressed and the floating columns before and after the stress. The change is indicated with the arrows.

\section{LATERAL ION TRANSPORT}

\section{A. Proof}

Using the method described above, from the measured transmission we derived the effective voltage over the LC (proportional to the ion concentration) as a function of the lateral position $x$ at different stress times (fig. 5).

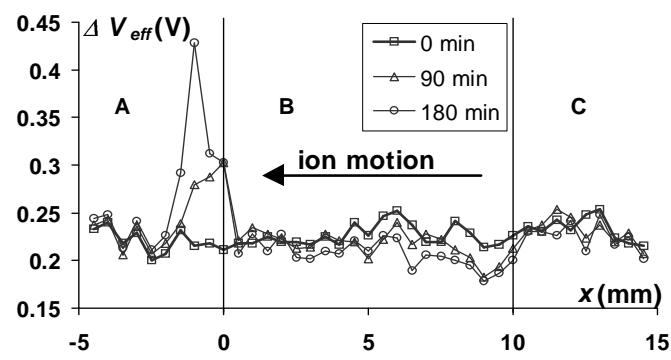

Fig. 5 Lateral distribution of the ion concentration (proportional to the $\Delta V_{\text {eff }}$. The ions move from right to left and pile-up after the edge of the column B

Here we can clearly see the evolution of the ion concentration along the lateral axis. Together with the electrical measurements before and after the stress (fig. 4), this is a direct proof that during the stress of $2 \mathrm{~V}_{\mathrm{ampl}}, 100 \mathrm{~Hz}$, the ions have moved laterally to the left (negative $x$ direction on the given figures). The fact that the cell turns brighter in the region with more ions is due to the ion effect on the effective voltage over the cell [5].

\section{B. Discussion}

The reason why the ions move perpendicular to the applied field is still unclear and is a subject of further study. However, using this phenomenon, we can explain the observed image retention. During the stress of the middle column B the ions travel towards its left edge. As soon as they enter the adjacent column A they stop, because the column is not stressed and the ion speed in it is zero. The ion concentration to the left of the edge increases rapidly as new ions arrive, because all the ions concentrate in a very narrow region. The piled-up ions diffuse to the left in the non-stressed column (A) contributing to the spreading of the 'dark band' (fig. 3). On the other side of the column, close to the edge B-C, the ions move to the left, and no new ions come from the right, because column $\mathrm{C}$ is not stressed. Therefore the ion concentration in the stressed column B left of the edge B-C decreases. With increasing the stress time, the brighter region (fewer ions) spreads to the left because ions gradually move away from it. This explanation corresponds well with the measured transmission (fig. 3) and the ion distribution (fig. 5).

The boundary image retention tends to disappear with time if the cell is not stressed. This is because of the diffusion of ions and the leveling-out of the ion concentration throughout the cell. Of course, since large distances are involved, the time necessary for the diffusion can be expressed in days.

\section{CONCLUSION}

When we stressed a column of our test cell for a longer time, image retention has occurred near the borders with the adjacent non-stressed columns. We have assumed that the origin of the boundary image retention is the lateral transport of ions. This assumption has been proven using a combination of electrical and optical measurements. The general trend of the measured lateral ion distribution agrees with our explanation of the ion motion. The physical cause of this phenomenon, however, still remains unknown. The mechanism and the conditions that lead to the lateral ion motion are subject to further investigations.

\section{ACKNOWLEDGEMENTS}

This work has been financially supported by Philips Research, Eindhoven, The Netherlands. ${ }^{1}$

\section{REFERENCES}

[1] C. Colpaert, B. Maximus, C. M. Groeneveld, IDRC 1994, pp. 191194

[2] N. Sasaki, Mol. Cryst. Liq. Cryst 367 (2001), pp. 671-679

[3] H. De Vleeschouwer, F. Bougrioua and H. Pauwels, Mol. Cryst. Liq. Cryst 360 (2001), pp. 29-39

[4] A. Lien, C.-J. Chen, H. Inove, Y. Saitoh, SID 1997, pp. 203-206

[5] A. Sawada, H. Sato, Y. Nakazono, S. Naemura, A. Manabe, SID 1999, pp. 190-193

\footnotetext{
1 This is a short version of the article accepted for publication in the proceedings of the $19^{\text {th }}$ ILCC 2002 in Molecular Crystals and Liquid Crystals.
} 